\title{
Ruptura parcial do tríceps distal em um atleta: Relato de caso e descrição de técnica cirúrgica*
}

\section{Partial Rupture of the Distal Triceps in an Athlete: Case Report and Description of Surgical Technique}

\author{
Eduardo Antônio Figueiredo ${ }^{1}$ Paulo Henrique Schmidt Lara10 Gyoguevara Patriota ${ }^{1}$ \\ Ronaldo Roncetti Júnior ${ }^{1}$ Paulo Santoro Belangero ${ }^{1}$ Benno Ejnisman ${ }^{1}$ \\ ${ }^{1}$ Departamento de Ortopedia e Traumatologia, Escola Paulista de \\ Medicina (EPM), Universidade Federal de São Paulo (Unifesp), \\ São Paulo, SP, Brasil

\begin{abstract}
Endereço para correspondência Paulo Henrique Schmidt Lara, MD, Departamento de Ortopedia e Traumatologia, Escola Paulista de Medicina (EPM), Universidade Federal de São Paulo (Unifesp),
\end{abstract} \\ São Paulo, SP, Brasil (e-mail: phslara@gmail.com).
}

Rev Bras Ortop 2019;54:601-604.

\author{
Resumo \\ Palavras-chave \\ - traumatismos em \\ atletas \\ - traumatismos dos \\ tendões \\ - ruptura
}

\begin{abstract}
\footnotetext{
Trabalho desenvolvido no Centro de Traumatologia do Esporte, Departamento de Ortopedia e Traumatologia, Escola Paulista de Medicina (EPM), Universidade Federal de São Paulo (Unifesp), São Paulo, SP, Brasil. Publicado Originalmente por Elsevier Editora Ltda.
}

A ruptura do tendão do tríceps braquial corresponde a menos de $1 \%$ de todas as rupturas tendíneas nos membros superiores. As rupturas parciais são subdiagnosticadas, o que pode fazer com que uma ruptura parcial se torne total. As rupturas completas geralmente requerem tratamento cirúrgico; entretanto, para rupturas parciais não há uma conduta bem definida. Este artigo apresenta o caso de um atleta de jiu-jitsu, do sexo masculino, de 42 anos, com ruptura parcial do tríceps braquial submetido a tratamento cirúrgico devido à persistência de perda de força de extensão do cotovelo, mesmo após tratamento conservador. Foi feito o reparo com enxerto do tendão do músculo palmar longo com técnica desenvolvida pelos autores. Não foram observadas complicações e o paciente apresentou resultado satisfatório, comprovado pela melhoria nos parâmetros dos estudos isocinéticos, os quais foram aferidos antes da cirurgia e com 5 meses de pós-operatório. Essa técnica demonstrou ser uma opção para casos de ruptura parcial do tríceps braquial em pacientes com alta demanda física que não apresentaram melhoria com o tratamento conservador.

Brachial triceps tendon ruptures account for less than $1 \%$ of all tendinous ruptures in the upper limbs. Partial ruptures are underdiagnosed, which may cause a partial rupture to become total. Complete ruptures usually require surgical treatment; however, for partial ruptures there is no well-defined conduct. This article presents the case of a 42-year-old male jiu-jitsu athlete with partial rupture of the brachial triceps who underwent surgical treatment due to persistent loss of elbow extension strength, even after conservative treatment. The repair was performed with grafting of the recebido

24 de Outubro de 2017

aceito

09 de Novembro de 2017
DOI https://doi.org/

10.1016/j.rbo.2017.11.007. ISSN 0102-3616.
Copyright $\odot 2019$ by Sociedade Brasileira License terms de Ortopedia e Traumatologia. Published by Thieme Revnter Publicações Ltda, Rio de Janeiro, Brazil 


\section{Keywords}

- athletic injuries

- tendon injuries

- rupture tendon of the long palmar muscle, utilizing a technique developed by the authors. No complications were observed, and the patient presented a satisfactory result, evidenced by the improvement in the parameters of isokinetic studies, which were performed before surgery and at 5 months postoperatively. This technique has proven to be an option for cases of partial rupture of the brachial triceps in patients with high physical demand who do not show improvement with the conservative treatment.

\section{Introdução}

Ruptura do tendão do tríceps braquial corresponde a menos de $1 \%$ de todas as rupturas tendíneas nos membros superiores, ${ }^{1}$ mas com o recente desenvolvimento e a prática de esportes de alta energia e de contato, houve um aumento na incidência de ruptura do tendão do tríceps braquial.

Rupturas parciais são subdiagnosticadas, o que pode fazer com que uma ruptura parcial se torne total. ${ }^{2}$ Rupturas completas geralmente requerem tratamento cirúrgico; ${ }^{3}$ entretanto, para rupturas parciais não há uma conduta bem definida.

\section{Relato de Caso}

Um paciente do sexo masculino, 42 anos, destro, professor de natação e praticante de jiu-jitsu. 0 paciente relatou que durante treino de jiu-jitsu recebeu um golpe na parte posterior do braço esquerdo e durante 7 dias apresentou dor no local. Após esse período, o paciente relatou melhoria do quadro de dor, porém perda de força para extensão do cotovelo, que o limitou para as atividades de natação e jiu-jitsu. Ele foi encaminhado ao nosso serviço após 3 meses sem melhoria com tratamento conservador.

Não havia alterações no exame clínico e a força muscular para extensão do cotovelo era similar ao lado contralateral.

As radiografias não apresentaram alterações. A ultrassonografia demonstrou acentuada tendinopatia distal do tríceps, com espessamento fusiforme de suas fibras ao longo de aproximadamente oito centímetros, com roturas intrassubstanciais e parciais de alto grau, distava cerca de quatro centímetros de sua inserção, comprometiam particularmente a cabeça longa, e em menor grau, a cabeça lateral.

Após 3 meses de fisioterapia supervisionada em nosso serviço, o paciente mantinha o quadro de perda de força para as atividades físicas mencionadas anteriormente. Frente a esse quadro, optamos pelo tratamento cirúrgico.

\section{Técnica Cirúrgica}

O paciente foi submetido a tratamento cirúrgico, no qual foi usado o acesso posterior clássico ( $\mathbf{- F i g . ~ 1 )}$ ). Foi constatada a integridade do tendão do tríceps, mas, a cerca de quatro centímetros da inserção do tríceps no olécrano, foi visualizada fibroseno tendão da cabeça longa e cabeça lateral, o que acreditamos ter se formado no local das rupturas parciais. 0 enxerto do tendão do músculo palmar longo foi retirado com quatro pequenas incisões transversais (-Fig. 2). Túneis transósseos foram criados no olécrano, com uma broca de $2,5 \mathrm{~mm}$. Proximalmente, o enxerto foi passado pelo tendão do tríceps com o ponto de Pulvertaft ( - Fig. 3). O enxerto foi passado com o cotovelo em flexão de 90 . O cotovelo foi mobilizado em todo o arco de movimento para garantir o tensionamento apropriado.

O paciente foi imobilizado por 14 dias com uma tala gessada axilopalmar. Depois desse período, iniciou reabilitação para ganho de arco de movimento.

\section{Avaliação Biomecânica}

O paciente foi avaliado através do dinamômetro isocinético (CybexII + , Nova York, NY, Estados Unidos) no pré-operatório e 5 meses após a cirurgia. Foi avaliada a flexoestensão. 0 braço direito foi usado como o lado controle.

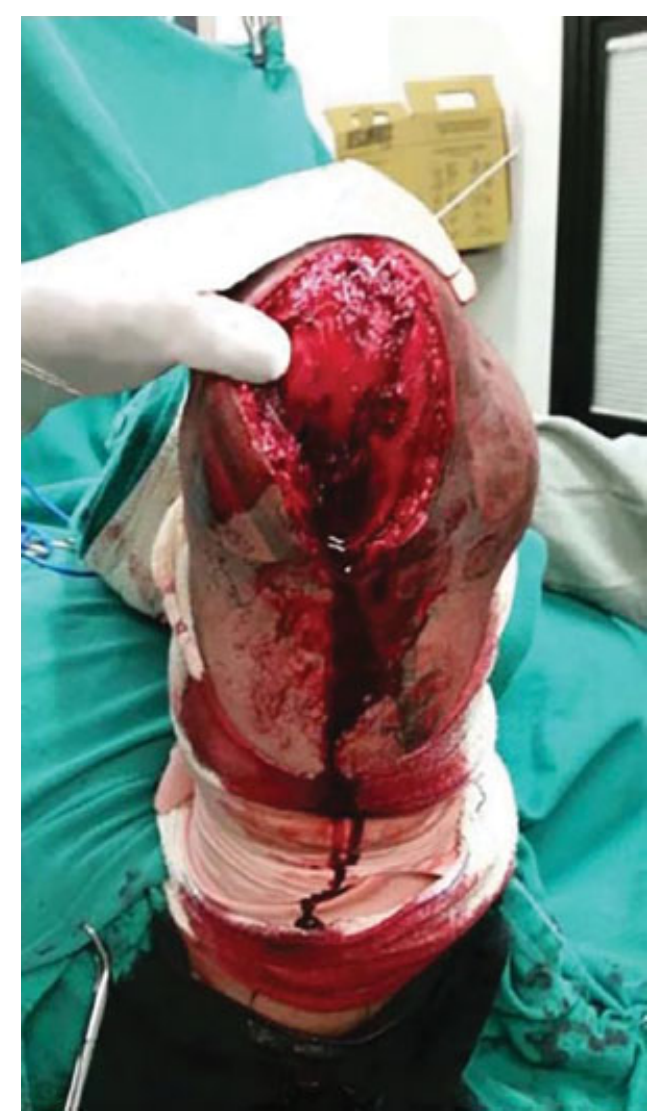

Fig. 1 Acesso posterior cirúrgico. 


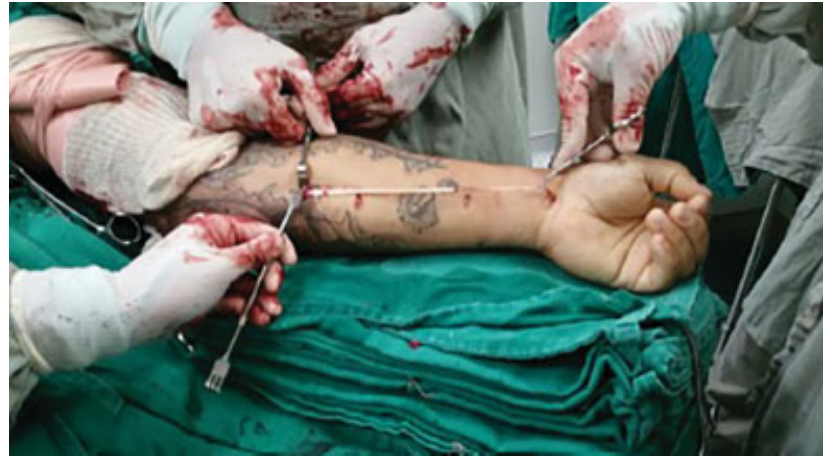

Fig. 2 Retirada do enxerto (tendão do musculo palmar longo).

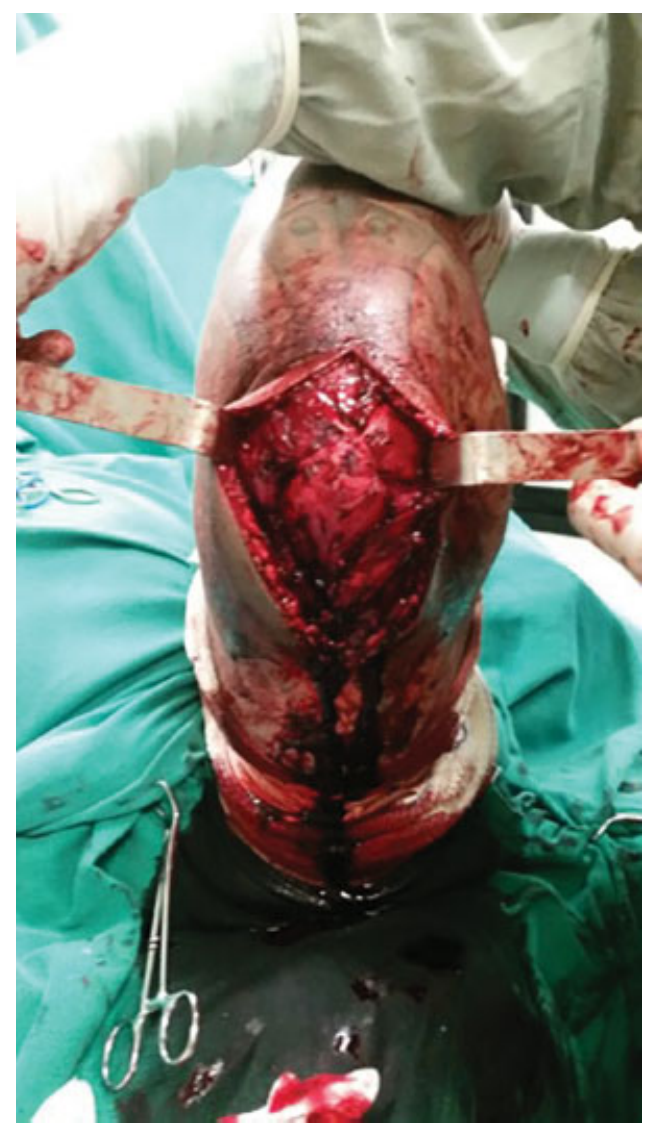

Fig. 3 Configuração final do reforço feito com enxerto do tendão do palmar longo.

\section{Avaliação de Forca}

Pico isocinético de força foi feito para flexoextensão do cotovelo em 60 e 300。 por segundo. 0 paciente foi estabilizado em posição supina, ombro em abdução de 45 o e o eixo do dinamômetro era alinhado com o epicôndilo umeral com o antebraço em rotação neutra. 0 arco de movimento do cotovelo foi estabilizado entre 30 e 135 o de flexão.

\section{Avaliação do Resultado Biomecânico}

Cinco meses após a cirurgia, o paciente apresentou melhoria dos valores de pico isocinético de força, apresentou diminuição dos déficits em relação ao lado controle tanto para extensão quanto para flexão. A 60。 o déficit de extensão em relação ao lado controle passou de $22 \%$ antes da cirurgia para 8,9\% 5 meses após cirurgia e a 300。 o déficit de extensão em relação ao lado controle passou de 24,8\% antes da cirurgia para 5,2\% 5 meses após a cirurgia. 0 paciente foi submetido a ultrassonografia do braço com 5 meses de pós-operatório, a qual demonstrou integridade do tríceps distal, sem sinais de roturas.

\section{Discussão}

O diagnóstico de rupturas parciais do tendão do tríceps pode ser difícil. Em algumas circunstâncias, um defeito é palpável proximal ao olécrano ou há apenas fraqueza para extensão do cotovelo. ${ }^{4}$ No caso apresentado, o paciente apresentava apenas perda de força de extensão do cotovelo que o prejudicava as atividades físicas. Vale ressaltar que se o paciente fosse um paciente com baixa demanda funcional, provavelmente não haveria necessidade do tratamento empregado neste caso.

Muitas técnicas foram descritas para a reconstrução do tendão do tríceps após rupturas completas ou parciais. Reparo primário de uma ruptura completa com sutura não absorvível com passagem em furos feitos no olécrano tem sido preconizado sempre que possível. ${ }^{5,6}$ o uso de autoenxerto ou aloenxerto tem sido preconizado quando o reparo primário não for possível. $^{6}$

Wagner et al. ${ }^{7}$ descreveram um avanço em V-Y do tendão do tríceps com reforço do reparo com enxerto do músculo plantar que foi suturado nas fáscias medial e lateral do antebraço.

Scolaro et al. ${ }^{8}$ descreveram dois casos de rupturas crônicas em que usaram como enxerto o tendão do palmar longo fixação proximal do enxerto pela técnica de Pulvertaft.

Khiami et al. ${ }^{9}$ descreveram um caso de ruptura parcial em que fizeram a reinserção da cabeça medial do tríceps no olécrano e essa parte ainda foi suturada ao restante do músculo. No estudo de Nikolaidou et al., ${ }^{10}$ foi feita a avaliação isocinética com 18 meses de pós-operatório em um paciente fisiculturista submetido a reconstrução de uma ruptura total do tríceps com enxerto sintético. $O$ paciente apresentou resultados superiores no lado operado em todos os parâmetros avaliados.

No caso em questão, usamos reforço com enxerto do tendão do palmar longo mesmo em uma ruptura parcial porque acreditamos que traria mais força para a reconstrução e tensão adequada e permitiria uma reabilitação mais precoce. O uso do enxerto do músculo palmar longo é uma boa opção, já que se situa num local próximo ao da cirurgia principal, a técnica para retirada é relativamente fácil, além de ter uma espessura pequena, o que permite que sejam confeccionados furos pequenos na ulna que minimizam o risco de complicações como fraturas.

Conflitos de Interesse

Os autores declaram não haver conflitos de interesse.

\section{Referências}

1 Anzel SH, Covey KW, Weiner AD, Lipscomb PR. Disruption of muscles and tendons; an analysis of 1, 014 cases. Surgery 1959;45 (03):406-414 
604 Ruptura Parcial do Tríceps Distal em Atleta Figueiredo et al.

2 Bennett BS. Triceps tendon rupture. Case report and a method of repair. J Bone Joint Surg Am 1962;44(04):741-744

3 Mair SD, Isbell WM, Gill TJ, Schlegel TF, Hawkins RJ. Triceps tendon ruptures in professional football players. Am J Sports Med 2004; 32(02):431-434

4 Herrick RT, Herrick S. Ruptured triceps in a powerlifter presenting as cubital tunnel syndrome. A case report. Am J Sports Med 1987; 15(05):514-516

5 van Riet RP, Morrey BF, Ho E, O'Driscoll SW. Surgical treatment of distal triceps ruptures. J Bone Joint Surg Am 2003;85(10): 1961-1967

6 Morrey BF. Rupture of the triceps tendon. In: Morrey BF, editor. The elbow and its disorders. Philadelphia: Saunders; 2008: 536-546
7 Wagner JR, Cooney WP. Rupture of the triceps muscle at the musculotendinous junction: a case report. J Hand Surg Am 1997; 22(02):341-343

8 Scolaro JA, Blake MH, Huffman GR. Triceps tendon reconstruction using ipsilateral palmaris longus autograft in unrecognized chronic tears. Orthopedics 2013;36(01):e117-e120

9 Khiami F, Tavassoli S, De Ridder Baeur L, Catonné Y, Sariali E. Distal partial ruptures of triceps brachii tendon in an athlete. Orthop Traumatol Surg Res 2012;98(02):242-246

10 Nikolaidou ME, Banke IJ, Laios T, Petsogiannis K, Mourikis A. Synthetic augmented suture anchor reconstruction for a complete traumatic distal triceps tendon rupture in a male professional bodybuilder with postoperative biomechanical assessment. Case Rep Orthop 2014;2014:962930 